\title{
Collateral Score Complements Clot Location in Predicting the Outcome of Intravenous Thrombolysis
}

\author{
J.T. Saarinen, H. Rusanen, and N. Sillanpää
}

\begin{abstract}
BACKGROUND AND PURPOSE: Collateral circulation is an important determinant of stroke outcome. We studied the impact of leptomeningeal collateral circulation with respect to the location of the thrombus in predicting the clinical outcome of patients treated with intravenous thrombolytic therapy ( $<3$ hours) in a retrospective cohort.
\end{abstract}

MATERIALS AND METHODS: Anterior circulation thrombus was detected with CT angiography in 105 patients. Baseline clinical and imaging information was collected, and the site of the occlusion was recorded. Collaterals were assessed by using a 5 -grade collateral score and were entered into logistic regression analysis to predict favorable clinical outcome (3-month modified Rankin Scale score of 0-2).

RESULTS: Two-thirds of patients with a proximal occlusion displayed poor collateral filling (collateral score $0-1$ ), whereas in more distal clot locations, approximately one-third had poor collaterals. Only $36 \%$ of patients with a proximal occlusion and good collaterals experienced favorable clinical outcome. In multivariate analysis, both clot location and collateral score were highly significant $(P=.003$ and $P=.001$ ) and independent predictors of favorable clinical outcome. Good collateral status increased the odds of favorable clinical outcome about 9-fold $(\mathrm{OR}=9.3 ; 95 \% \mathrm{Cl}, 2.4-35.8)$. After dichotomization, a distal clot location had a larger odds ratio (OR $=13.3 ; 95 \% \mathrm{Cl}$, $3.0-60.0)$ compared with the odds ratio of good collaterals ( $\mathrm{OR}=5.9 ; 95 \% \mathrm{Cl}, 1.8-19.0)$.

CONCLUSIONS: A proximal occlusion in the anterior circulation is associated with poorer collateral status compared with a more distal occlusion. Both the clot location and collateral score are important and independent predictors of favorable clinical outcome of hyperacute stroke treated with intravenous thrombolysis. The location of the clot is a stronger determinant of the outcome than the collateral score.

ABBREVIATIONS: $\mathrm{CS}=$ collateral score; HIS = hyperacute ischemic stroke; IVT = intravenous thrombolytic therapy; MID = distal M1 segment of the MCA; MIP = proximal M1 segment of the MCA

I schemic stroke results from occlusion of an artery, with subsequent reduction in regional cerebral blood flow. The ischemic penumbra can remain viable for hours because some degree of blood flow is sustained through the leptomeningeal collateral supply. An important aspect of the work-up of patients with acute neurovascular syndrome is imaging of cervical and intracranial vasculature to detect the location of the occluding clot and evaluate the integrity of the collateral circulation. Both of these potentially influence decision-making for revascularization thera-

Received January 22, 2014; accepted after revision March 23.

From the Department of Neurology (J.T.S.), University of Tampere, Tampere and Vaasa Central Hospital, Vaasa, Finland; Department of Neurology (H.R.), Oulu University Hospital, Oulu, Finland; and Medical Imaging Center (N.S.), Tampere University Hospital, Tampere, Finland.

This work was supported by the Tampere University Hospital governmental subsidiary funds for clinical research.

Please address correspondence to Niko Sillanpää, MD, PhD, Medical Imaging Center, Tampere University Hospital, PL 2000, 33521, Tampere, Finland; e-mail: niko. sillanpaa@pshp.fi

http://dx.doi.org/10.3174/ajnr.A3983 pies. Patients with proximal occlusions have a poor prognosis, even if treated with intravenous thrombolytic therapy (IVT). ${ }^{1} \mathrm{~Pa}-$ tients with good collateral status have larger penumbra ${ }^{2}$ and respond better to both IVT and intra-arterial interventions, ${ }^{2-5}$ whereas diminished or absent collaterals are associated with increased stroke severity, faster progression, and worse outcome. ${ }^{6-8}$ Unfortunately, it appears that patients with a more proximal clot more frequently have worse collateral status. ${ }^{9}$

The purpose of our study was to analyze the impact of leptomeningeal collateral circulation with respect to the location of the clot in predicting 24-hour imaging findings and the 3-month clinical outcome of hyperacute ischemic stroke (HIS) in patients treated with IVT ( $<3$ hours). We discuss the interplay between the location of the clot and the collateral circulation.

\section{MATERIALS AND METHODS Study Population}

Our retrospective observational cohort study was approved by the Tampere University Hospital ethics committee. Altogether 315 
patients with anterior or posterior circulation HIS were treated with IVT from January 2004 to December 2007 and had a 3-month follow-up after thrombolysis at the department of neurology of the Tampere University Hospital. CT angiography had been performed at admission in 285 (90\%) of these patients. CTA was not performed because of previously known contrast agent hypersensitivity, chronic renal failure, or imminent closure of the 3-hour time window. Inclusion criteria for the study were acute anterior circulation vessel occlusion confirmed with CTA and treatment with a standard IVT administration protocol. The thrombolytic therapy protocol used was in line with the American Heart Association guidelines. ${ }^{10}$

\section{Participants and Variables}

Baseline clinical characteristics were collected from patient records. The National Institutes of Health Stroke Scale score at the time of administration of rtPA had been prospectively stored. Follow-up noncontrast-enhanced CT and NIHSS scoring were performed for all patients 24 hours after the administration of the thrombolytic agent. The modified Rankin Scale score 3 months after the stroke was the primary outcome measure. The 3-month $\mathrm{mRS}$ score was prospectively recorded on the basis of a follow-up visit to a neurologist or a phone interview by a neurologist.

\section{Imaging Parameters}

CT scans were obtained by using 2 different multidetector scanners: LightSpeed 16-detector row (GE Healthcare, Milwaukee, Wisconsin) and Brilliance 64-detector row (Philips Healthcare, Best, the Netherlands). CTA was performed from the C2 vertebra to the vertex. The imaging parameters were the following: $120 \mathrm{kV}$; $212 \mathrm{mAs}$ (dynamic tube-current modulation); collimation, $64 \times$ $0.625 \mathrm{~mm}$; rotation, 0.75 seconds; pitch factor, 0.923 (64-detector row); or $120 \mathrm{kV}$; $160 \mathrm{mAs}$; collimation, $16 \times 0.625 \mathrm{~mm}$; rotation, 0.8 seconds; pitch factor, 0.938 (16-detector row). Contiguous sections were reconstructed to 0.9 -mm thickness by using 0.45 -mm overlap (64-detector row) or 1.25-mm thickness (16-detector row). The contrast agent (iobitridol, Xenetix, $350 \mathrm{mg} \mathrm{I/mL;} \mathrm{Guerbet,} \mathrm{Aulnay-sous-}$ Bois, France) was administered through an antecubital 18-ga cannula by using a double-piston power injector with a flow rate of 4 $\mathrm{mL} / \mathrm{s}$ by using $70 \mathrm{~mL}$ of contrast agent followed by a $50-\mathrm{mL}$ saline flush. Manual bolus triggering was used. NCCT was performed as described in our previous report. ${ }^{11}$

\section{Image Analysis}

The Alberta Stroke Program Early CT Score was assessed from admission and follow-up NCCT images, and CTA studies were interpreted as described in our previous article. ${ }^{11}$ Infarcts with a volume of $\leq 10 \mathrm{~mL}$ in the 24-hour NCCT were defined as minor infarcts and those $\geq 100 \mathrm{~mL}$ were considered extensive infarcts. The location of the clot was recorded on the basis of the most proximal position of the occlusion. The M1 segment of the middle cerebral artery was divided into 2 parts of equal length: the proximal and the distal halves (designated as M1P and M1D). The status of the leptomeningeal collateral circulation was evaluated by using the scoring system devised by Souza et al. ${ }^{8}$ In short, the collateral score (CS) was determined from MIP images according to the following rules: $0=$ absent collaterals in $>50 \%$ of an M2 branch territory; $1=$ diminished collaterals in $>50 \%$ of an M2 branch territory; $2=$ diminished collaterals in $<50 \%$ of an M2 branch territory; 3 = collaterals equal to the contralateral hemisphere; and $4=$ increased collaterals.

The clot location was determined and CS was scored independently by 2 radiologists. In cases in which the scoring or the assignment differed, a consensus opinion was agreed on. The intraclass correlation coefficient between a staff radiologist and an experienced neuroradiologist for a test sample $(n=20)$ for CS was 0.87 . Cohen $\kappa$ was 0.94 for the location of the clot and 0.68 for the CS (0.90 after dichotomization).

\section{Statistics}

The data were analyzed with the Statistical Package for the Social Sciences, Version 19 (IBM, Armonk, New York). Group comparisons were performed by using the Student $t$ test, the $\chi^{2}$ test, the Fisher exact test, the Mann-Whitney $U$ test, and the Kruskal-Wallis test. Patients with collateral scores from 2 to 4 had good collateral vessel filling. Patients who had 3 -month $\mathrm{mRS} \leq 2$ had favorable clinical outcome. Binary logistic regression modeling by using this outcome measure as the dependent variable was repeated for single covariates of interest and their combinations. An odds ratio with a $95 \% \mathrm{CI}$ was calculated for each covariate. The calibration of the models was evaluated with the Hosmer-Lemeshow test, and the discrimination, with the $\mathrm{C}$ statistic.

\section{RESULTS}

\section{Baseline Characteristics}

We studied 105 consecutive patients who met the inclusion criteria: acute anterior circulation vessel occlusion followed by IVT. The demographic and baseline characteristics of the study population have been described in depth in our previously published work. ${ }^{1}$ The main baseline and other characteristics are summarized in Table 1. Fifty-four (52\%) patients experienced favorable clinical outcome $(\mathrm{mRS} \leq 2)$ at 3 months. One patient could not be reached with telephone or by other means for evaluation of mRS. Thirty-eight (36\%) patients had a proximally located (distal ICA and/or proximal half of the M1 segment of MCA) occlusion, and $58(55 \%)$ patients had good collateral status (CS 2-4). Overall, 29 (28\%), 18 (17\%), 20 (19\%), $36(34 \%)$, and 2 (2\%) patients had CSs of $0,1,2,3$, and 4 , respectively. The differences in baseline characteristics between patients with good and poor collateral status, proximal and distal occlusions, and favorable and unfavorable clinical outcome are given in Table 1. Poor collateral circulation was associated with more severe strokes according to admission NIHSS (15 versus $11, P=.02$ ), more extensive ischemic changes in the admission NCCT $(P<.001)$, worse clinical and imaging outcomes 24 hours after the treatment $(P<.001)$, and somewhat shorter onset-to-treatment times (124 minutes versus 138 minutes, $P=.008$ ).

\section{Collateral Score and Clinical Outcome in Different Clot Locations}

The distribution of CSs in different clot locations is depicted in Fig 1 , where CS 3-4 has been pooled into 1 group. In the 2 most proximal clot locations (ICA and M1P), approximately twothirds of the patients (59\% and 66\%, respectively) displayed poor 
Table 1: Demographic and baseline characteristics of all patients and by good collateral status, the locus of the thrombus, and 3-month outcome

\begin{tabular}{|c|c|c|c|c|c|c|c|c|c|c|}
\hline Characteristic & $\begin{array}{l}\text { All Patients } \\
(n=105)\end{array}$ & $\begin{array}{c}\text { Collateral } \\
\text { Score 0-1 } \\
(n=47)\end{array}$ & $\begin{array}{c}\text { Collateral } \\
\text { Score 2-4 } \\
(n=58)\end{array}$ & $\mathbf{P}_{1}$ & $\begin{array}{c}\mathrm{mRS} \leq 2 \\
\text { at Day } 90 \\
(n=54)\end{array}$ & $\begin{array}{c}\text { mRS 3-6 } \\
\text { at Day 90 } \\
(n=50)\end{array}$ & $\mathbf{P}_{2}$ & $\begin{array}{c}\text { Proximal } \\
\text { Thrombus } \\
\text { (ICA+MIP, } \\
n=38)\end{array}$ & $\begin{array}{c}\text { Distal } \\
\text { Thrombus } \\
\text { (M1D+M2+M3, } \\
n=67)\end{array}$ & $\mathbf{P}_{3}$ \\
\hline Age (yr) (mean) (SD) & $68.8(13.5)$ & $70.1(14.4)$ & $67.8(12.7)$ & .38 & 66.4 (13.1) & $71.3(13.6)$ & .06 & $66.0(15.1)$ & $70.4(12.3)$ & .11 \\
\hline Male (\%) & $60(57)$ & $29(62)$ & $31(53)$ & .40 & $32(59)$ & $28(56)$ & .74 & 27 (71) & $33(49)$ & .03 \\
\hline $\begin{array}{l}\text { NIHSS score before treatment } \\
\text { (median) (IQR) }\end{array}$ & $13(10)$ & $15(7)$ & $11(12)$ & .02 & $9(10)$ & $17(7)$ & $<.001$ & $18(7)$ & $11(10)$ & $<.001$ \\
\hline $\begin{array}{l}\text { NIHSS score } 24 \text { hours after } \\
\text { thrombolysis (median) (IQR) }\end{array}$ & $6(14)$ & $14(14)$ & $3(7)$ & $<.001$ & $2(4)$ & $16(11)$ & $<.001$ & $15(10)$ & $3(7)$ & $<.001$ \\
\hline $\begin{array}{l}\text { ASPECTS at admission CT } \\
\text { (median) (IQR) }\end{array}$ & $10(2)$ & $8(3)$ & $10(0)$ & $<.001$ & $10(1)$ & $9(2)$ & .20 & $9(3)$ & $10(2)$ & .07 \\
\hline $\begin{array}{l}\text { ASPECTS at } 24 \text { hours after } \\
\text { CT (median) (IQR) }\end{array}$ & $7(5)$ & $5(4)$ & $8.5(3)$ & $<.001$ & $9(3)$ & $5(4)$ & $<.001$ & $4(5)$ & $8(4)$ & $<.001$ \\
\hline $\begin{array}{l}\text { Collateral score (median) } \\
\text { (IQR) }\end{array}$ & $2(3)$ & $0(1)$ & $3(1)$ & $<.001$ & $3(1)$ & $1(2)$ & $<.001$ & $1(3)$ & $2(2)$ & .01 \\
\hline $\begin{array}{l}\text { Onset-to-treatment time } \\
\text { (min) (mean) (SD) }\end{array}$ & $132(27)$ & $124(26)$ & $138(27)$ & .008 & $133(26)$ & $129(29)$ & .46 & $131(31)$ & $132(25)$ & .85 \\
\hline Hypertension (No.) (\%) & $69(65.7)$ & $31(66)$ & $38(66)$ & .96 & $36(66.7)$ & $33(66.0)$ & .94 & $22(57.9)$ & $47(70.1)$ & .20 \\
\hline Diabetes (No.) (\%) & $17(16.2)$ & 9 (19) & $8(14)$ & .46 & $6(11.1)$ & $11(22.0)$ & .13 & $8(21.1)$ & $9(13.4)$ & .31 \\
\hline Atrial fibrillation (No.) (\%) & $41(39.0)$ & $19(40)$ & $22(38)$ & .79 & $23(42.6)$ & $18(36.0)$ & .49 & $12(31.6)$ & $29(43.3)$ & .24 \\
\hline $\begin{array}{l}\text { Coronary artery disease } \\
\text { (No.) (\%) }\end{array}$ & $35(33.3)$ & $19(40)$ & $16(28)$ & .17 & $12(22.2)$ & $23(46.0)$ & .01 & $16(42.1)$ & $19(28.4)$ & .15 \\
\hline
\end{tabular}

Note: $-P_{1}$ indicates a $P$ value between poor and good collateral status; $P_{2}, P$ value between $m R S \leq 2$ and mRS $>2$ groups; $P_{3}, P$ value between the proximal and distal thrombus groups; IQR, interquartile range; min, minutes.

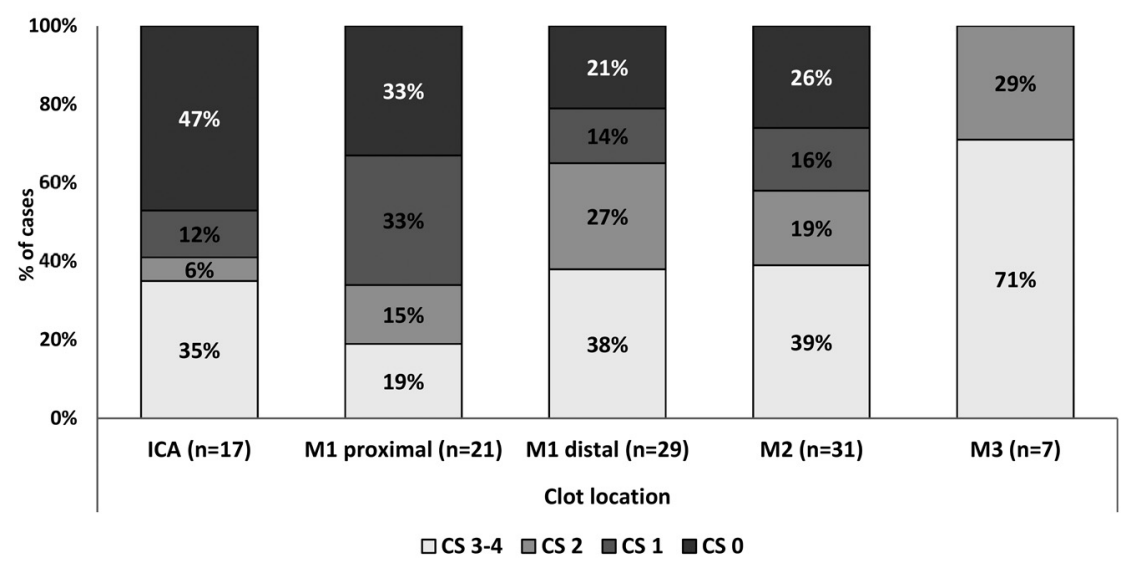

FIG 1. The distribution of collateral scores in different clot locations. Collateral scores 3 and 4 are pooled into same group.

(CS $0-1)$ collateral filling, whereas in the 2 more distal clot locations (M1D and M2), only about one-third (35\% and 42\%, respectively) had poor collaterals. In the most distant clot location studied (M3), all patients had good collateral status. The distribution of the CS was significantly different across the studied clot locations $(P=.04)$. When adjoining clot locations (ICA-M1P, M1P-M1D, M1D-M2, M2-M3) were compared in pairs, only the difference in the distribution of CS between M1P and M1D yielded statistical significance $(P=.05)$.

To assess the prognostic value of CS in different clot locations, we dichotomized the CS as described above and cross-tabulated it with the dichotomized 3-month mRS score $(\mathrm{mRS} \leq 2)$ in different clot locations. Overall, poor collateral status was associated with unfavorable clinical outcome, especially in the proximal clot locations: Not a single patient with acute occlusion of the ICA and having poor collaterals experienced favorable clinical outcome $(P<.001)$. However, the association between good collaterals and favorable clinical outcome was less pronounced in the proximal locations, 29\% in ICA and 43\% in the M1P. When individual clot locations were considered, only M1D showed statistically significant differences with $70 \%$ of those with poor collaterals experiencing unfavorable outcome and $74 \%$ of those with good collaterals experiencing favorable outcome $(P=.05)$.

On the basis of these results, location was dichotomized by using M1P-M1D as the dividing point and cross-tabulation was repeated. A proximal clot was more strongly associated with unfavorable outcome than poor collateral status (Fig 2). However, good collaterals were associated with improved outcome in both proximal and distal clot locations $(P=.08$ and $P=$ .004, respectively).

To further assess the prognostic value of CS and its interplay with the clot location, we performed binary logistic regression analysis by using the dichotomized 3-month mRS score as the dependent variable. The CS was analyzed with the model we used in our previous article. ${ }^{1}$ When the site of the occlusion was included in the model as a covariate, onset-to-treatment times, sex, diabetes, hypertension, atrial fibrillation, and coronary heart disease, tested one at a time, were not statistically significant covariates. Age, NIHSS score, CS, sex, and onset-totreatment times were kept in the final multivariate regression model. The latter 2 variables were included for theoretic reasons, though they did not reach statistical significance in the preliminary analysis. The resulting model (Table 2 ) displayed satisfying fit and calibration (Hosmer-Lemeshow test, $P=.95$; C statistic $=$ 0.92). The model was also tested with an interaction term (CS* clot location) that proved not to be statistically significant. Both the clot location and the CS were highly significant $(P=.003$ and $P=.001$, respectively) independent predictors of favorable clinical outcome in the presence of the NIHSS score (Table 2). Good collateral status increased the odds of favorable clinical out- 


\section{distal clot and good collaterals $(n=43)$ \\ distal clot and poor collaterals $(n=23)$}

proximal clot and good collaterals
\[ (\mathrm{n}=14) \]

proximal clot and poor collaterals $(n=24)$

$16 \%$ $84 \%$

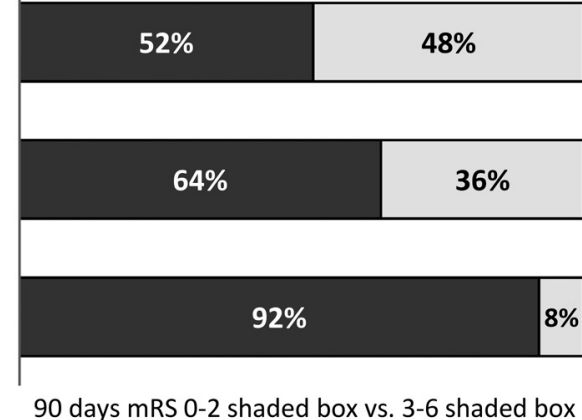

mRS at 90 days $3-6 \quad \square \mathrm{mRS}$ at 90 days 0 - 2

FIG 2. Collateral score and the site of the occlusion predict the clinical outcome. A proximal clot (ICA or MIP) is more strongly associated with unfavorable outcome than poor collateral status (CS $0-1)$.

Table 2: Logistic regression analysis for favorable clinical outcome $^{\mathrm{a}}$

\begin{tabular}{lccc}
\hline & \multicolumn{3}{c}{ mRS $\leq 2$ at 2 Months } \\
\cline { 2 - 4 } & Odds Ratio & $\mathbf{9 5 \%}$ Cl & P Value \\
\hline Clot location & - & - & .003 \\
ICA & ref & ref & - \\
M1 proximal & 10.1 & $0.74-140$ & .08 \\
M1 distal & 33.8 & $2.9-428$ & .007 \\
M2 and M3 & 115.8 & $7.7-1737$ & .001 \\
Onset-to-treatment time & 0.99 & $0.97-1.02$ & .44 \\
Sex & 0.34 & $0.09-1.3$ & .11 \\
Age & 0.95 & $0.90-0.99$ & .02 \\
Admission NIHSS score & 0.81 & $0.71-0.92$ & .001 \\
Favorable CS (2-4) & 9.3 & $2.4-35.8$ & .001 \\
\hline
\end{tabular}

Note:-ref indicates reference location; -, not applicable.

a Odds ratios are per minute for onset-to-treatment time, per year for age, and per 1 point for NIHSS.

come about 9-fold (OR $=9.3 ; 95 \% \mathrm{CI}, 2.4-35.8)$. The OR for favorable clinical outcome increased in a graded fashion when moving from a proximal vessel position to a more distal one. A higher NIHSS score and advanced age were significantly associated with worse outcome. Finally, clot location was dichotomized similar to that in Fig 2 (cut-point at M1P/M1D), and the model was recalculated. A distal clot location had a larger odds ratio $(\mathrm{OR}=13.3 ; 95 \% \mathrm{CI}, 3.0-60.0)$ compared with that of good collateral status $(\mathrm{OR}=5.9 ; 95 \% \mathrm{CI}, 1.8-19.0)$.

\section{Collateral Score Identifies Patients at Risk of Developing an Extensive Infarct}

When cross-tabulated with dichotomized CS, 79\% of patients with minor infarcts $(\leq 10 \mathrm{~mL})$ in the 24-hour follow-up NCCT had good collaterals, whereas $61 \%$ of patients with larger-thanminor infarcts $(>10 \mathrm{~mL})$ had poor collateral circulation $(P<$ $.001)$. Good collateral circulation was associated with minor infarcts, especially in the distal clot positions. Ninety-two percent of patients with a clot in the M1D and a minor infarct had good collaterals $(P=.02) ; 72 \%$ of patients with M2 occlusion and a minor infarct had good collaterals $(P=.08)$. Some patients $(17 \%$, 18 of 105) had an extensive infarct ( $\geq 100 \mathrm{~mL})$. Two-thirds (12 of
18) of these had a proximal (ICA/M1P) occlusion. Most (89\%, 16 of 18$)$ patients with an extensive infarct had poor collaterals according to the CS $(P<.001)$.

\section{DISCUSSION}

We studied the interplay between the location of the clot and the collateral status with regard to the 3-month clinical and the 24-hour imaging outcomes in a HIS cohort treated with IVT.

The tendency of patients with more proximal thrombi and larger clot burden to have poorer collateral status has been observed recently. ${ }^{9}$ In our study, the proportion of patients with HIS with good collateral status doubled when the location of the occlusion moved from the proximal half of the M1 segment to the distal half. When the clot was found in the M3 segment, the collateral status was always good; this outcome can be expected from the definition of CS based on vascular territories supplied by the M2 segment arteries. The differential distribution of the CS in different clot locations may be due to proximal thrombi and poor collaterals sharing common risk factors, such as advanced atherosclerosis, old age, and hypertension. ${ }^{12,13}$ However, an obvious mechanism explaining this observation is that the more proximal the occlusion and the more extensive the volume of the ischemic brain parenchyma and the more profound the reduction of cerebral blood flow in the ischemic core, the more easily is the capacity of the collateral vessels overwhelmed, resulting in a lower CS.

In the multivariate analysis, both the clot location and the CS proved to be highly significant and independent predictors of favorable clinical outcome, a finding that is in line with previous studies. ${ }^{1-3,5-8,14-17}$ The cohorts of these studies were heterogeneous as to the use and types of revascularization therapies and onset-to-treatment times. Two of these studies analyzed cohorts consisting exclusively of patients undergoing IVT and/or intraarterial thrombolysis, and they found that a high degree of collateralization predicted a good response to IVT. ${ }^{3,5}$ However, these studies did not include the location of the clot as a covariate in their multivariate models, or the location of the clot was not a statistically significant predictor. By using a multivariate model, Lima et $\mathrm{al}^{15}$ found that both the site of the intracranial occlusion and the pattern of leptomeningeal collateral circulation predicted the functional outcome of patients with anterior circulation stroke when all treatment modalities were considered. When only patients without revascularization therapy were analyzed, the site of the occlusion was not a significant determinant. In our cohort, adding the CS to a model already containing the location of the clot and NIHSS score resulted in better model fit (C statistic, 0.92 versus 0.90 ). Good collateral status increased the odds of favorable clinical outcome about 9-fold, and the odds of favorable clinical outcome increased substantially when the clot location was more distal. The site of the occlusion proved to be a stronger determinant of the outcome; good collaterals combined with IVT 
managed to save only about one-third (36\%) of the patients with a proximal clot from functional dependence or death at 3 months.

Poor collateral circulation is a major risk factor for already having developed an extensive infarct volume at admission. ${ }^{8}$ The combination of a proximal thrombus and poor collaterals is referred to as a "malignant profile." 8 Supporting this concept, in our study, $89 \%$ of patients who had an extensive $(>100 \mathrm{~mL})$ infarct in the 24-hour follow-up NCCT had poor collateral filling. These findings emphasize the importance of timely and correct therapeutic decision-making in this patient subgroup and may have a role in avoiding futile recanalization. ${ }^{18}$

Selection bias related to the retrospective design is a potential limitation of this study. Direct data on vessel recanalization or reperfusion were not available. Even so, a low ASPECTS at 24hour NCCT is intimately related to delayed or failed recanalization/reperfusion and can be used as a surrogate. CTA has limitations in the evaluation of collateral circulation. It provides a snapshot of the filling of collaterals at the time of image acquisition. It has been shown that this may lead to underestimation of the collateral circulation because of late vessel filling. ${ }^{19}$ Finally, the impact of the CS or clot location on clinical outcome may vary according to treatment variables beyond consideration of this study, such as rehabilitation or withdrawal of care after severe stroke.

\section{CONCLUSIONS}

The results of this study show that a proximal site of occlusion in the anterior circulation is associated with poorer collateral status compared with a more distal occlusion. Both the location of the clot and the CS are important, independent predictors of the 3-month clinical outcome in the context of HIS treated with IVT. The location of the clot is a more powerful determinant of clinical outcome than the CS. However, poor collateral circulation is closely associated with extensive infarct volumes.

\section{ACKNOWLEDGMENTS}

We thank Jari Hakomäki, MD, for image interpretation.

Disclosures: Jukka T. Saarinen-UNRELATED: Payment for Lectures (including service on Speakers Bureaus): Boehringer Ingelheim. Niko Sillanpää—RELATED: Grant: Pirkanmaa Hospital District Clinical Studies Fund, grant number 9N070.

\section{REFERENCES}

1. Saarinen JT, Sillanpaa N, Rusanen H, et al. The mid-M1 segment of the middle cerebral artery is a cutoff clot location for good outcome in intravenous thrombolysis. Eur J Neurol 2012;19:1121-27

2. Miteff F, Levi CR, Bateman GA, et al. The independent predictive utility of computed tomography angiographic collateral status in acute ischaemic stroke. Brain 2009;132:2231-38

3. Calleja AI, Cortijo E, Garcia-Bermejo P, et al. Collateral circulation on perfusion-computed tomography-source images predicts the response to stroke intravenous thrombolysis. Eur J Neurol 2013; 20:795-802

4. Christoforidis GA, Mohammad Y, Kehagias D, et al. Angiographic assessment of pial collaterals as a prognostic indicator following intra-arterial thrombolysis for acute ischemic stroke. AJNR Am J Neuroradiol 2005;26:1789-97

5. Kucinski T, Koch C, Eckert B, et al. Collateral circulation is an independent radiological predictor of outcome after thrombolysis in acute ischaemic stroke. Neuroradiology 2003;45:11-18

6. Bang OY, Saver JL, Buck BH, et al. Impact of collateral flow on tissue fate in acute ischaemic stroke. J Neurol Neurosurg Psychiatry 2008;79:625-29

7. Maas MB, Lev MH, Ay H, et al. Collateral vessels on CT angiography predict outcome in acute ischemic stroke. Stroke 2009;40:3001-05

8. Souza LC, Yoo AJ, Chaudhry ZA, et al. Malignant CTA collateral profile is highly specific for large admission DWI infarct core and poor outcome in acute stroke. AJNR Am J Neuroradiol 2012; 33:1331-36

9. Tan IY, Demchuk AM, Hopyan J, et al. CT angiography clot burden score and collateral score: correlation with clinical and radiologic outcomes in acute middle cerebral artery infarct. AJNR Am J Neuroradiol 2009;30:525-31

10. Adams HP Jr, Brott TG, Furlan AJ, et al. Guidelines for thrombolytic therapy for acute stroke: a supplement to the guidelines for the management of patients with acute ischemic stroke-a statement for healthcare professionals from a Special Writing Group of the Stroke Council, American Heart Association. Circulation 1996; 94:1167-74

11. Sillanpaa N, Saarinen JT, Rusanen H, et al. CT perfusion ASPECTS in the evaluation of acute ischemic stroke: thrombolytic therapy perspective. Cerebrovasc Dis Extra 2011;1:6-16

12. Arsava EM, Vural A, Akpinar E, et al. The detrimental effect of aging on leptomeningeal collaterals in ischemic stroke. J Stroke Cerebrovasc Dis 2014;23:421-26

13. Shuaib A, Butcher K, Mohammad AA, et al. Collateral blood vessels in acute ischaemic stroke: a potential therapeutic target. Lancet Neurol 2011;10:909-21

14. Bang OY, Saver JL, Kim SJ, et al. Collateral flow predicts response to endovascular therapy for acute ischemic stroke. Stroke 2011; 42:693-99

15. Lima FO, Furie KL, Silva GS, et al. The pattern of leptomeningeal collaterals on CT angiography is a strong predictor of long-term functional outcome in stroke patients with large vessel intracranial occlusion. Stroke 2010;41:2316-22

16. Menon BK, Smith EE, Modi J, et al. Regional leptomeningeal score on CT angiography predicts clinical and imaging outcomes in patients with acute anterior circulation occlusions. AJNR Am J Neuroradiol 2011;32:1640-45

17. Sillanpää N, Saarinen JT, Rusanen H, et al. Location of the clot and outcome of perfusion defects in acute anterior circulation stroke treated with intravenous thrombolysis. AJNR Am J Neuroradiol 2013;34:100-06

18. Liebeskind DS, Sanossian N. How well do blood flow imaging and collaterals on angiography predict brain at risk? Neurology 2012;79:S105-09

19. Smit EJ, Vonken EJ, van Seeters T, et al. Timing-invariant imaging of collateral vessels in acute ischemic stroke. Stroke 2013;44:2194-99 